\title{
Key Preassigned Traceability Schemes for Broadcast Encryption
}

\author{
D. R. Stinson and R. Wei \\ Department of Combinatorics and Optimization \\ University of Waterloo \\ Waterloo Ontario, N2L 3G1, Canada
}

\begin{abstract}
Traceability schemes for broadcast encryption are defined by Chor, Fiat and Naor in 6 to protect against a possible coalition of users producing an illegal decryption key. Their scheme was then generalized by Stinson and Wei in 17 . These schemes assume that every user can decrypt the secret value. In this paper we discuss key preassigned traceability schemes, in which only the users in a specified privileged subset can decrypt. A new scheme is presented in this paper, which has better traceability than previous schemes. We also present a new threshold traceability scheme by using ramp scheme. All the constructions are explicit and could be implemented easily.

Keywords: key preassigned scheme, broadcast encryption, traceability, secret sharing schemes, combinatorial designs.
\end{abstract}

\section{Introduction}

Most networks can be thought of as broadcast networks, in that any one connected to the network can access to all the information that flows through it. In many situations, such as a pay-per-view television broadcast, the data is only available to authorized users. To prevent an unauthorized user from accessing the data, the trusted authority (TA) will encrypt the data and give the authorized users keys to decrypt it. Some unauthorized users might obtain some decryption keys from a group of one or more authorized users (called traitors). Then the unauthorized users can decrypt data that they are not entitled to. To prevent this, Chor, Fiat and Naor 6 devised a traitor tracing scheme, called a traceability scheme, which will reveal at least one traitor on the confiscation of a pirate decoder. This scheme was then generalized by Stinson and Wei in 17 . There are some other recent papers discussing this topic (see 10 12 1.3).

The basic idea of a traceability scheme is as follows. Suppose there are a total of $b$ users. The TA generates a set $T$ of $v$ base keys and assigns $\ell$ keys chosen from $T$ to each user. These $\ell$ keys comprise a user's personal key, and we will denote the personal key for user $i$ by $U_{i}$. A broadcast message, $M$, consists of an enabling block, $B$, and a cipher block, $Y$. The cipher block is the encryption of the actual plaintext data $X$ using a secret key, $S$. That is, $Y=e_{S}(X)$, where $e(\cdot)$ is the encryption function for some cryptosystem. The enabling block consists of data which is encrypted by some method, using some or all of the $v$ keys in

S. Tavares and H. Meijer (Eds.): SAC'98, LNCS 1556, pp. 144- 1999.

(C) Springer-Verlag Berlin Heidelberg 1999 
the base set, the decryption of which will allow the recovery of the secret key $S$. Every authorized user should be able to recover $S$ using his or her personal key, and then decrypt the cipher block using $S$ to obtain the plaintext data, i.e., $X=d_{S}(Y)$, where $d(\cdot)$ is the decryption function for the cryptosystem.

Some traitors may conspire and give an unauthorized user a pirate decoder, $E$. $E$ will consist of a subset of base keys such that $E \subseteq \cup_{i \in C} U_{i}$, where $C$ is the coalition of traitors. An unauthorized user may be able to decrypt the enabling block using a pirate decoder. The goal of the TA is to assign keys to the users in such a way that when a pirate decoder is captured and the keys it possesses are examined, it should be possible to detect at least one traitor in the coalition $C$, provided that $|C| \leq c$ (where $c$ is a predetermined threshold).

In all the traceability schemes discussed in 6171012 it is assumed that every user can decrypt the enabling block. This means that the data supplier should assign the keys after he or she has determined who the authorized users are. In practice, however, this restriction may be inconvenient, as changes between authorized and unauthorized users may be frequent.

In this paper, we investigate traceability schemes in which the personal keys can be assigned before the authorized users are determined. We will call these schemes key preassigned schemes. Key preassigned schemes (for broadcast encryption) have been discussed by several researchers. The first scheme was introduced by Berkovits in II. Several recent papers have studied broadcast encryption schemes (see $34 \times 1315 \mathrm{I0}$, for example). Broadcast schemes enable a TA to broadcast a message to the users in a network so that a certain specified subset of authorized users can decrypt it. However, most of these broadcast schemes have not considered the question of traceability. We will briefly review the traceability of these schemes and then give some key preassigned schemes which have better traceability than the previous schemes. We will also discuss threshold tracing schemes which are more efficient but less secure in some respect. We will use combinatorial methods to describe the schemes and give some explicit constructions. The efficiency of the schemes is measured by considering the information rate and broadcast information rate.

There are two aspects of security in our schemes. One property of the scheme is to prevent unauthorized users from decrypting the enabling block; this is the usual question investigated in broadcast encryption. The second property is the ability of tracing a pirate decoder which is made by a coalition of users (which of course could be authorized users). Although these two properties both protect against coalitions, they have different effects. The first property can prevent the coalition of unauthorized users from decrypting the enabling block, but it does not protect against construction of a pirate decoder. The second property cannot prevent a coalition from decrypting the enabling block, but it enables the TA to trace at least one traitor if the decoder is found.

We will discuss unconditionally secure (in an information theoretic sense) schemes. These schemes do not depend on any computational assumption. 


\section{Definitions and notations}

In this section, we give basic definitions and the notations used in this paper.

\subsection{Broadcast encryption schemes}

The definition of a broadcast encryption scheme we use in this paper will be the same as the one given in $I .5$. As in a traceability scheme, there is a trusted authority (TA) and a set of users $\mathcal{U}=\{1,2, \cdots, b\}$, and the TA generates a set of $v$ base keys and assigns a subset of the base keys to each user as his or her personal key. At a later time, a privileged subset, $P$, of authorized users is determined. The TA chooses a secret key $S$ and broadcasts an enabling block $B_{P}$ (which is an encryption of $S$ ) that can be decrypted by every authorized user, but which cannot be decrypted by certain forbidden subsets disjoint from $P$.

Let $\mathcal{P}$ denote the collection of possible privileged subsets and let $\mathcal{F}$ denote the collection of possible forbidden subsets. In this paper, we will consider the case when $\mathcal{P}=2^{\mathcal{U}}$, so $\mathcal{P}$ contains all subsets of users, and $\mathcal{F}$ contains all $f$ subsets of users, where $f$ is a fixed integer. To make things simpler (and since we want to focus on the traceability first), we will mainly consider the situation when $f=1$. In the case $\mathcal{P}=2^{\mathcal{U}}$ and $f=1$, the privileged subset can be chosen to be any subset of users, and the enabling block cannot be decrypted by an individual unauthorized user. (It may be possible for subsets of unauthorized users to jointly decrypt the message, however.)

For $1 \leq i \leq b$, let $\mathbf{U}_{i}$ denote the set of all possible subsets of base keys that might be distributed to user $i$ by the TA. Thus the personal key $U_{i} \in \mathbf{U}_{i}$. Let $\mathbf{S}$ denote the set of possible secret keys, so $S \in \mathbf{S}$. Let $\mathbf{B}_{P}$ be the set of possible enabling blocks for privileged subset $P$; thus $B_{P} \in \mathbf{B}_{P}$. Usually, $U_{i}, S$ and $B_{P}$ consist of tuples from a finite field $\mathbb{F}_{q}$. We define the information rate to be

$$
\rho=\min \left\{\frac{\log \mathbf{S}}{\log \mathbf{U}_{i}}: 1 \leq i \leq b\right\} .
$$

and the broadcast information rate to be

$$
\rho_{B}=\min \left\{\frac{\log \mathbf{S}}{\log \mathbf{B}_{P}}: P \in \mathcal{P}\right\} .
$$

In general, to decrease the size of the broadcast, i.e., to increase $\rho_{B}$, it is necessary to decrease $\rho$, and vice versa. Since it is trivial to construct a broadcast encryption scheme with $\rho=1$ and $\rho_{B}=1 / b$, we are mainly interested in schemes with $\rho_{B}>1 / b$.

\subsection{Traceability}

Suppose a "pirate decoder" $E$ is found. (We assume that the pirate decoder can be used to decrypt some enabling blocks.) If there exists a user $i$ such that $\left|E \cap U_{i}\right| \geq\left|E \cap U_{j}\right|$ for all users $j \neq i$, then $i$ is defined to be an exposed user. A $c$-traceability scheme is defined as follows. 
Definition 21 Suppose any exposed user $i$ is a member of the coalition $\mathcal{C}$ whenever a pirate decoder $\mathrm{E}$ is produced by $\mathcal{C}$ (so $E \subseteq \cup_{i \in C} U_{i}$ ) and $|\mathcal{C}| \leq c$. Then the scheme is called a c-traceability scheme.

When a scheme is $c$-traceable, $\mathcal{P}=2^{\mathcal{U}}$, and the forbidden subsets consist of all $f$-subsets of users, we call it a $(c, f)$-key preassigned traceability scheme and denote it as a $(c, f)$-KPTS. For the case $f=1$, we denote the scheme as a $c$-KPTS.

Remark. The difference between Definition 21 and the one in 13 is that the size of the pirate decoder is not specified here. For example, the pirate decoder might be smaller or larger than a legitimate decoder. The only requirement is that a pirate decoder should be able to decode some enabling blocks.

A set system is a pair $(X, \mathcal{A})$, where $X$ is a set of points and $\mathcal{A}$ is a collection of subsets of $X$ called blocks. We will use set systems with the following property, which is modified from 17 Theorem 2.2].

Definition 22 A traceability scheme system is a set system $(X, \mathcal{A})$, where every block has size $k$ for some integer $k$, with the property that for every choice of $c^{\prime} \leq c$ blocks $A_{1}, A_{2}, \cdots, A_{c^{\prime}} \in \mathcal{A}$, and for any $t$-subset $E \subseteq \cup_{j=1}^{c^{\prime}} A_{j}$, where $t \geq k$, there does not exist a block $A \in \mathcal{A} \backslash\left\{A_{1}, A_{2}, \cdots, A_{c^{\prime}}\right\}$ such that $\left|E \cap A_{j}\right| \leq|E \cap A|$ for $1 \leq j \leq c^{\prime}$. Such a system will be denoted by $(c, k)$-TSS.

In this definition, the blocks correspond to legitimate decoders and $E$ corresponds to a pirate decoder. We will be able to assume that $|E| \geq k$ due to the encryption scheme we use.

\subsection{Secret sharing schemes}

Let $\mathcal{U}$ be the set of $b$ users, $\Gamma \subseteq 2^{\mathcal{U}}$ be a set of subsets called authorized subsets, and let $\Delta \subseteq 2^{\mathcal{U}}$ be a set of subsets called unauthorized subsets. In a $(\Gamma, \Delta)$-secret sharing scheme, the TA has a secret value $K$. The TA will distribute secret information called shares to each user of $\mathcal{U}$ in such a way that any authorized subset can compute $K$ from the shares they jointly hold, but no unauthorized subset has any information about $K$. The paper 14 contains an introduction to secret sharing schemes.

Let $r<t \leq b$. An $(r, t, b)$-ramp scheme is a secret sharing scheme in which the authorized subsets are all the subsets of $\mathcal{U}$ with cardinality at least $t$ and the unauthorized subsets are all the subsets of $\mathcal{U}$ with cardinality at most $r$. When $r=t-1$, the ramp scheme becomes a threshold scheme which is denoted by $(t, b)$-threshold scheme. The Shamir scheme provides a construction of a $(t, b)$ threshold scheme in which each share is an element of $\mathbb{F}_{q}$ and the secret is also an element of $\mathbb{F}_{q}$, for any prime power $q \geq b+1$. 


\subsection{Key predistribution schemes}

Fiat-Naor key predistribution schemes (or KPS) (see 8 ) are used in the KIO construction for broadcast encryption schemes given in 15 . Let $f \leq b$ be an integer. The forbidden subsets consist of all subsets of size at most $f$. In a FiatNaor scheme, the TA chooses a secret value $x_{F}$ for each possible forbidden subset $F$, and gives that value to each user in $\mathcal{U} \backslash F$. Let $P \subseteq \mathcal{U}$. The value

$$
K_{P}=\sum_{F \cap P=\emptyset} x_{F}
$$

is the key for the privileged subset $P . K_{P}$ can be computed by any member of $P$, but $K_{P}$ cannot be computed by any forbidden subset $F$ disjoint from $P$ (where $|F| \leq f)$.

\section{Traceability of previous broadcast schemes}

Since key preassigned broadcast encryption schemes were proposed in I , several constructions have been given. A summary of these results can be found in Stinson 15. In 15, the KIO construction is described, and which is further discussed in 16 . We will not review these schemes here — we only wish to indicate that these schemes usually do not have any traceability, or have, at most, 1-traceability. (However, note that if in a scheme, every user has disjoint keys, then the scheme is "totally traceable". Thus the trivial scheme in 1.5 has b-traceability.)

Staddon first discussed the traceability of key preassigned broadcast schemes in her PhD thesis I.3. She constructed some schemes called "OR protocols" that have higher traceability. We briefly review the OR protocols now. In OR protocols, the size of a forbidden subset is $f$ and the size of the privileged subset is $w=b-f$. These values are fixed ahead of time. The TA produces a key $K_{t}$ for each subset $P_{t}$ of $\mathcal{U}$, where $\left|P_{t}\right|=\left\lceil\frac{w}{n}\right\rceil$, and gives that key to every user in $P_{t}$, where $n$ is a given positive integer. When the TA wants to broadcast an enabling block for a privileged subset $P$, he uses the $n$ keys in the set

$$
\mathcal{L}_{P}=\left\{K_{t}: P_{t} \subseteq P\right\}
$$

to encrypt it, in such a way that any user who has at least one of these $n$ keys is able to decrypt it.

It is shown in 13 that the OR protocol construction has $\Theta(\sqrt{n})$-traceability for $n>2$ and $b$ sufficiently large relative to $n$ and $f$. However, the proof is based on the assumption that the pirate decoder always is the same size as a personal key, i.e., that it always contains

$$
\left(\begin{array}{c}
b-1 \\
\left\lceil\frac{w}{n}\right\rceil-1
\end{array}\right)
$$

keys. This assumption may not be practical. In fact, unauthorized users who possess even one key might be able to decrypt the enabling block if the key 
happened to belong to the set $\mathcal{L}_{P}$. Thus the OR protocol has no traceability if we consider the traceability under Definition 21 where we allow a pirate decoder to have fewer keys than a personal key.

The traceability schemes in 617 have the desirable property that any possible decoder must consist of the keys from the base key set, otherwise they will be useless for decoding. In some other proposed schemes, an enabling block can be decrypted using keys not in the base set. In such a scheme, the traceability property is defeated. We describe the traceability scheme proposed in 10 to illustrate this point.

In the scheme of 10 (which is not key preassigned), the TA chooses a random polynomial

$$
f(x)=a_{0}+a_{1} x+a_{2} x^{2}+\cdots+a_{c} x^{c} .
$$

The TA then computes $f(i)$ and gives it to user $i$ secretly, so that the personal key of user $i$ will be $(i, f(i))$. When TA wants to encrypt the secret key $S$, he broadcasts the enabling block $\left(S+a_{0}, a_{1}, a_{2}, \cdots, a_{c}\right)$. If a pirate decoder contains a pair $(u, f(u))$, then $u$ will be the exposed user. However, two users $i$ and $j$ can construct a pirate decoder as follows. They choose two random non-zero numbers $\alpha$ and $\beta$ and compute the following:

$$
b_{0}=\frac{\alpha f(i)+\beta f(j)}{\alpha+\beta}, b_{1}=\frac{\alpha i+\beta j}{\alpha+\beta}, \cdots, b_{c}=\frac{\alpha i^{c}+\beta j^{c}}{\alpha+\beta} .
$$

Since

$$
a_{0}=b_{0}-a_{1} b_{1}-\cdots-a_{c} b_{c},
$$

the $(c+1)$-tuple $\left(b_{0}, \ldots, b_{c}\right)$ can be used as a decoder. In this scenario, the traitors $i$ and $j$ cannot be exposed by the usual traitor tracing method.

\section{The new scheme}

In this section, we present our traceability schemes which will use a KIO type construction. The basic idea of the $\mathrm{KIO}$ construction is that the secret key is split into shares, using a threshold scheme (or a ramp scheme), and then the shares are encrypted, thus forming the enabling block. Our scheme is a key preassigned broadcast encryption scheme where $\mathcal{U}=\{1, \ldots, b\}, \mathcal{P}=2^{\mathcal{U}}$ and $\mathcal{F}$ consists of all $f$-subsets of $\mathcal{U}$. We consider the case $f=1$ first.

Suppose $(X, \mathcal{A})$ is a $(c, k)$-TSS, where $X=\{1,2, \cdots, v\}$ and $\mathcal{A}=\left\{A_{1}, A_{2}, \cdots\right.$, $\left.A_{b}\right\}$. The block $A_{j}$ determines the personal key given to user $j$, for $1 \leq j \leq b$. For each $u \in X$, let

$$
R_{u}=\left\{j \in \mathcal{U}: u \in A_{j}\right\}
$$

The main steps in the protocol are as follows:

1. For every set $R_{u}$ as defined above, the TA constructs a Fiat-Naor key predistribution scheme on user set $R_{u}$, with $\mathcal{F}_{u}=\left\{\{j\}: j \in R_{u}\right\} \cup\{\emptyset\}$ and $\mathcal{P}_{u}=2^{R_{u}}$. Thus, for each $u, 1 \leq u \leq v$, the TA chooses $\left|R_{u}\right|+1$ secret 
values, denoted $x_{R_{u}}$ and $x_{R_{u}, j}\left(j \in R_{u}\right)$. These values are chosen at random from a finite field $\mathbb{F}_{q}$. The value $x_{R_{u}}$ is given to each $i \in R_{u}$ and $x_{R_{u}, j}$ is given to each $i \in R_{u} \backslash\{j\}$. These keys form the personal key for user $i$.

We will assume the existence of a function Index on the set of base keys such that $\operatorname{Index}(x)=j$ if $x$ is a key from the $j$ th Fiat-Naor scheme. These keys might be stored as pairs, e.g., $\left(x_{R_{u}}, u\right)$ and $\left(x_{R_{u}, j}, u\right)$, so that the users know which keys are from which Fiat-Naor scheme.

2. Suppose the TA wants to encrypt the secret key $S \in \mathbb{F}_{q}$ for a privileged subset $P$. For the purposes of illustration, suppose $P=\{1,2, \cdots, w\}$. The TA first uses a $(k, n)$-threshold scheme to split $S$ into $n$ shares $y_{1}, y_{2}, \cdots, y_{n}$, where $A_{P}=\cup_{i=1}^{w} A_{i}$ and $n=\left|A_{P}\right|$ (note that $n \leq v$, so a $(k, v)$ )-threshold scheme can be used here, if desired).

3. For each $j \in A_{P}$, the TA computes the secret key $K_{j}$ of Fiat-Naor scheme on $R_{j}$ for the privileged subset $R_{j} \cap P$, i.e.,

$$
K_{j}=x_{R_{j}}+\sum_{i \in R_{j} \backslash P} x_{R_{j}, i} .
$$

4. Each share $y_{j}$ is encrypted using an encryption function $e(\cdot)$ with key $K_{j}$. The enabling block consists of the list of encrypted values

$$
\left(e_{K_{j}}\left(y_{j}\right): j \in A_{P}\right) .
$$

Since each user in $P$ has $k$ values in $A_{P}$, he can compute $k$ keys $K_{i_{1}}, K_{i_{2}}, \cdots$, $K_{i_{k}}$ and then obtain $k$ shares, $y_{i_{1}}, y_{i_{2}}, \cdots, y_{i_{k}}$. Using the reconstruction function of the threshold scheme, the user is able to recover the value of the secret key, $S$.

A user not in $P$ cannot compute any of the keys $K_{i}$, since the Fiat-Naor scheme is secure against individual unauthorized users. Thus, the user cannot get any information about the $n$ shares.

Now we consider traceability. Suppose a pirate decoder $E$ is found. The TA can compute the Index of the decoder as

$$
\operatorname{Index}(E)=\{\operatorname{Index}(x): x \in E\} .
$$

Note that the cardinality of the set $\operatorname{Index}(E)$ is at least $k$, otherwise the decoder will be useless. The TA can then use this Index to find an exposed user, since the set system $(X, \mathcal{A})$ is a $(c, k)$-TSS.

The information rate of this scheme is

$$
\rho=\frac{1}{k r}
$$

where $r_{x}$ is the number of blocks containing $x$, i.e., $r_{x}=\left|R_{x}\right|$, and $r=\max \left\{r_{x}\right.$ : $x \in X\}$. The broadcast information rate is

$$
\rho_{B}=\frac{1}{n} \geq \frac{1}{v}
$$


The following theorem summarizes the properties of the scheme.

Theorem 41 Suppose $(X, \mathcal{A})$ is a $(c, k)$-TSS in which $|X|=v$ and $|\mathcal{A}|=b$. Then there is a $c$-KPTS for a set of $b$ users, having information rate $\rho \geq 1 /(k r)$ and broadcast information rate $\rho_{B} \geq 1 / v$.

Remark. For the case $f>1$, we need only change the construction of the FiatNaor scheme on each $R_{u}$ so that the possible forbidden subsets are all subsets of $R_{u}$ having size at most $f$. This will cause the information rate of the scheme to decrease, while the broadcast information rate remains the same.

The following small example will illustrate the scheme.

Example 41 A 2-KPTS with 82 users.

Let $X=\{0,1, \cdots, 40\}$ and suppose $\mathcal{A}$ contains the following 82 blocks, where the calculations are in $\mathbb{Z}_{41}$, for $i=0,1,2, \cdots, 40$ :

$$
\begin{aligned}
& A_{i}=\{1+i, 10+i, 18+i, 16+i, 37+i\} \\
& A_{41+i}=\{36+i, 32+i, 33+i, 2+i, 20+i\}
\end{aligned}
$$

The set system $(X, \mathcal{A})$ is a $(41,5,1)$-balanced incomplete block design (see 7$)$. This set system has the property that each pair of points appears in exactly one block, and every point appears in exactly 10 blocks. It is in fact a $(2,5)$-TSS (see Theorem 0.3 .

The block $A_{i}$ is associated with user $i$. For each $u \in X$, the TA constructs a Fiat-Naor scheme on $R_{u}$. For example, for $u=1$, it can be seen that

$$
R_{1}=\{0,32,24,26,5,47,51,50,81,63\},
$$

so $\left|R_{1}\right|=11$. The $\mathrm{TA}$ will choose 11 secret values in $\mathbb{F}_{q}$ for some prime power $q$, and every user in $R_{1}$ will receive 10 of the 11 values. A Fiat-Naor scheme is implemented in this way on each $R_{u}$, and thus every user has 50 values in his or her personal key.

Now, suppose the TA wants to encrypt a secret key $S \in \mathbb{F}_{q}$, where the privileged subset is $P=\{0,1,2, \cdots, 59\}$, so $w=60$. The TA uses a $(5,41)$ threshold scheme to split $S$ into 41 shares, $y_{0}, \ldots, y_{40}$. For example,

$$
K_{1}=x_{R_{1}}+x_{R_{1}, 63}+x_{R_{1}, 81} .
$$

The enabling block will be the list of encrypted values

$$
\left(e_{K_{0}}\left(y_{0}\right), \cdots, e_{K_{40}}\left(y_{40}\right)\right) \text {. }
$$

Any user in $P$ can decrypt the enabling block. For example, consider user 5 . The block $B_{5}=\{6,15,23,21,42\}$. Then user 5 obtains five of the 41 secret keys, namely, $K_{6}, K_{15}, K_{23}, K_{21}$ and $K_{42}$, and recovers the five shares $y_{6}, y_{15}, y_{23}$, $y_{21}$ and $y_{42}$. From these five shares $S$ can be obtained. 
Any user not in $P$ cannot decrypt the enabling block. For example, let us consider user 63 . If $j \notin B_{63}$, then user 63 does not have $K_{R_{j}}$ and cannot compute $K_{j}$. On the other hand, if $j \in B_{63}$, then user 63 does not have $K_{R_{j}, 63}$ and cannot compute $K_{j}$ either. Thus user 63 cannot compute any of the shares in the threshold scheme.

Finally, let's show that the scheme is 2-traceable. If a pirate decoder $E$ is found, then the TA can compute Index $(E)$ as described above. Index $(E)$ must contain at least 5 numbers, otherwise it cannot decode anything. Suppose that the decoder was made by two users, say $i$ and $j$. Since $\operatorname{Index}(E) \subseteq\left(B_{i} \cup B_{j}\right)$ it must be the case that $\left|\operatorname{Index}(E) \cap B_{i}\right| \geq 3$ or $\left|\operatorname{Index}(E) \cap B_{j}\right| \geq 3$. Since any two blocks intersect in at most one point, $\left|\operatorname{Index}(E) \cap B_{h}\right| \leq 2$ if $\bar{h} \neq i, j$. Thus user $i$ or user $j$ (or both) will be exposed users.

\section{Threshold tracing}

In the schemes of Section $n$ the Index of any pirate decoder should contain at least $k$ values, otherwise the decoder cannot get any information from the broadcast. However, as indicated in II (the final version of 6 ), such security is not needed in many applications. For example, in pay-TV applications pirate decoders which decrypt only part of the content are probably useless. Thus II defined the concept of a threshold traceability scheme. In a threshold traceability scheme, the tracing algorithm only can trace the decoders which decrypt with probability greater than some threshold $p$. In this section, we discuss some key preassigned threshold traceability schemes, denoted by KPTTS. Our approach is quite different from the methods used in 11. We will use ramp schemes to construct KPTTS.

We can obtain a ramp scheme from an orthogonal array.

Definition 51 An orthogonal array $\mathrm{OA}(t, k, s)$ is an $s^{t} \times k$ array, with entries from a set $Y$ of $s \geq 2$ symbols, such that in any $t$ columns, every $t \times 1$ row vector appears exactly once.

The following lemma ( $a$ Chapter VI.7]) provides infinite classes of orthogonal arrays, for any integer $t$.

Lemma 51 If $q$ is a prime power and $t<q$, then there exists an $O A(t, q+1, q)$.

Suppose there is an $\mathrm{OA}(t, v+t-r, q)$ which is public knowledge. The secret information $K$ is a $(t-r)$-tuple from $\mathbb{F}_{q}$. The TA chooses secretly a row in the OA such that the last $t-r$ columns of that row contains the tuple $K$. It is easy to see that there are $q^{r}$ such rows. The TA then gives each of the $v$ users one value from the first $v$ columns of that row. Since any $t$ of these values determine a row of the OA uniquely, $t$ users can get $K$ by combining their shares. However, from any $r$ values, the users cannot obtain any information about $K$, since these $r$ values together with last $t-r$ columns of any row in the OA determine that 
row. (For more detailed description of this construction, the reader can consult 9 .

Our KPTTS is similar to the KPTS constructed in Section 4 The only difference is that we use a $(0, k, n)$-ramp scheme to split the message into shares in a KPTTS, instead of the $(k, n)$-threshold scheme used in the KPTS.

In the KPTTS, the base key set and preassigned keys are the same as in the KPTS. However, when the TA wants to send a secret message $M \in\left(\mathbb{F}_{q}\right)^{k}$ to a privileged subset, the TA uses a $(0, k, n)$-ramp scheme to split $M$ into $n$ shares. The TA uses the same method of KPTS to encrypt the $n$ values, and broadcasts the resulting list of $n$ values. Similar to the KPTS, any user in the privileged subset can compute $k$ keys, so he or she can recover the $n$ values from the ramp scheme, but the users not in the privileged subset cannot get any information from the encryption.

Now suppose that a pirate decoder $E$ is found. If the size of $\operatorname{Index}(E)$ is not less than $k$, then the TA can find an exposed user as he did in the KPTS. When the size of Index $(E)$ is less than $k$, the TA may not be able to trace the users in the coalition. So let us see what a decoder $E$ could do, if the $\operatorname{Index}(E)$ contains $k-1$ values. Note that the ramp scheme is constructed from an $\mathrm{OA}(k, k+v, q)$. For any $k-1$ values, there are $q$ rows which contain these $k-1$ values. Among these $q$ rows, only one row carries the secret message $M$. Hence the decoding threshold of the KPTTS is

$$
p=\frac{1}{q} .
$$

The information rate of the KPTTS is the same as that of the KPTS, but the broadcast information rate of the KPTTS is much better. In the KPTTS, we have

$$
\rho_{B}=\frac{k}{n} \geq \frac{k}{v}
$$

Similar to the KPTS, the KPTTS is also based on the set systems TSS. We will discuss the construction of TSS in the next section.

\section{Constructions of traceability set systems}

To construct our traceability schemes, we need to find traceability set systems. Some constructions for these types of set systems were given in $\mathrm{I7}$; they are based on certain types of combinatorial designs. (A comprehensive source for information on combinatorial designs is Colbourn and Dinitz 7 .) We present a useful lemma for constructing TSS, and mention some applications of it.

Lemma 61 Suppose there exists a set system $(X, \mathcal{A})$ satisfying the following conditions:

1. $|A|=k \geq c^{2} \mu+1$ for any $A \in \mathcal{A}$;

2. $\left|A_{i} \cap A_{j}\right| \leq \mu$ for any $A_{i}, A_{j} \in \mathcal{A}, i \neq j$.

Then the set system is a $(c, k)$-TSS. 
Proof. Let $E \subseteq \cup_{i=1}^{c} A_{i}$ with $|E| \geq k$. Since $k \geq c^{2} \mu+1$, there is a block $A_{s}$, $1 \leq s \leq c$, such that $\left|E \cap A_{s}\right| \geq c \mu+1$. For any $A \in \mathcal{A} \backslash\left\{A_{1}, A_{2}, \cdots A_{c}\right\}$, we have

$$
\begin{aligned}
|E \cap A| & \leq\left|A \cap\left(\cup_{i=1}^{c} A_{i}\right)\right| \\
& \leq c \mu \\
& <c \mu+1 \\
& \leq\left|E \cap A_{s}\right| .
\end{aligned}
$$

Hence, the set system is a $(c, k)$-TSS.

As a first application of Lemma 61 we give a construction using $t$-designs.

Definition $61 A t$ - $(v, k, \lambda)$ design is a set system $(X, \mathcal{A})$, where $|X|=v$ and $|A|=k$ for all $A \in \mathcal{A}$, such that every $t$-subset of $X$ appears in exactly $\lambda$ blocks of $\mathcal{A}$.

Theorem 62 Suppose there exists a $t$ - $(v, k, 1)$ design. Then there exists a $(c, k)$ TSS, where $c=\lfloor\sqrt{(k-1) /(t-1)}\rfloor$.

Proof. Any two blocks of a $t$ - $(v, k, 1)$ design intersect in at most $t-1$ points. Apply Lemma bl with $\mu=t-1$.

There are many results on $t$ - $(v, k, 1)$ designs for small values of $t$, i.e., for $2 \leq t \leq 6$. See 7 for a summary of known results. We can construct interesting TSS using designs with $t=2$. For example, it is known that there is a $2-(v, 5,1)$ design for all $v \geq 5, v \equiv 1,5 \bmod 20$. These designs give rise to an infinite family of $(2,5)$-TSS. Applying Theorem 41 we have the following KPTS.

Theorem 63 There exists a 2-KPTS for all $v \geq 5, v \equiv 1,5 \bmod 20$, for a set of $b=v(v-1) / 20$ users, having $\rho=\frac{4}{5(v-1)}$ and $\rho_{B}=\frac{1}{v}$.

Note that Example 4 il is the case $v=41$ of the above theorem.

Similarly, we have

Theorem 64 There exists a 2-KPTTS for all $v \geq 5, v \equiv 1,5 \bmod 20$, for a set of $b=v(v-1) / 20$ users, having $\rho=\frac{4}{5(v-1)}$ and $\rho_{B}=\frac{5}{v}$.

A 3- $\left(q^{2}+1, q+1,1\right)$ design, known as an inversive plane, exists for any prime power $q$. The following result concerns the KPTS and KPTTS that can be constructed from inversive planes.

Theorem 65 For any prime power $q$, there exist a c-KPTS and a c-KPTTS, where $c=\left\lfloor\sqrt{\frac{q}{2}}\right\rfloor$, with information rate $\rho \approx \frac{1}{q^{3}}$ and broadcast information rates $\rho_{B} \approx \frac{1}{q^{2}}$ for KPTS and $\rho_{B} \approx \frac{1}{q}$ for KPTTS. 
In 11 , it is proved that there exists a threshould traceability scheme with broadcast information rate $\rho_{B}=O\left(\frac{1}{4 c}\right)$. However, the proof of that is not explicit. Our construction is explicit and the threshold of our scheme is usually better than that of the scheme in 11. Also our scheme is key preassigned.

Many other constructions of TSS can be given using combinatorial objects such as packing designs, orthogonal arrays, universal hash families, etc. The constructions are similar to those found in 1617 .

\section{Some remarks}

We make a couple of final observations in this section.

- The $(c, f)$-KPTS scheme discussed in this paper is a generalization of the traceability schemes in $6 \Gamma 7$. The schemes in $5 \Gamma 7$ are in fact the case of $f=0$ of our main construction. When $f=0$, there is no protection against an unauthorized user decrypting the enabling block.

- Most broadcast schemes and traceability schemes in the literature are described as unconditionally secure schemes. If the encryption function $e(\cdot)$ used in the scheme in this paper is addition in a finite field $\mathbb{F}_{q}$, then our scheme is also unconditionally secure. However, the drawback of using the above unconditionally secure encryption scheme is that the resulting KPTS and KPTTS will be a one-time scheme. On the other hand, if we desire only computational security, then we can replace $e(\cdot)$ by any cryptosystem that is computationally secure against a known plaintext attack, and we will obtain a KPTS that can be used for many broadcasts. This simple modification can be applied to other one-time schemes described in previously published papers.

\section{Acknowledgment}

The authors' research is supported by the Natural Sciences and Engineering Research Council of Canada. We would also like to acknowledge Tran van Trung for helpful discussions concerning this research.

\section{References}

1. S. Berkovits, How to broadcast a secret, Advances in Cryptology: EUROCRYPT'91, Lecture Notes in Computer Science, 547 (1992), 536-541.

2. J. Bierbrauer, T. Johansson, G. Kabatianskii and B. Smeets, On families of hash functions via geometric codes and concatenation, Advances in Cryptology - CRYPTO'93, Lecture Notes in Computer Science, 773 (1994), 331-342.

3. C. Blundo, and A. Cresti, Space requirement for broadcast encryption, Advances in Cryptology: EUROCRYPT'94, Lecture Notes in Computer Science, 950 (1995), 287-298. 
4. C. Blundo, L.A. Frota Mattos and D.R. Stinson, Trade-offs between communication and storage in unconditionally secure schemes for broadcast encryption and interactive key distribution, Advances in Cryptology: CRYPTO'96, Lecture Notes in Computer Science, 1109 (1996), 387-400.

5. J. L. Carter and M. N. Wegman, Universal classes of hash functions, J. Computer and System Sci., 18 (1979), 143-154.

6. B. Chor, A. Fiat and M. Naor, Tracing traitors, Advances in Cryptology: CRYPTO'94, Lecture Notes in Computer Science 839 (1994), 257-270.

7. C.J. Colbourn and J.H. Dinitz, eds., CRC Handbook of Combinatorial Designs, CRC Press, Inc., 1996.

8. A. Fiat and M. Naor, Broadcast encryption, Advances in Cryptology: CRYPTO'93, Lecture Notes in Computer Science, 773 (1994), 480-491.

9. W-A. Jackson and K. M. Martin, A combinatorial interpretation of ramp schemes, Austral. J. Combinatorics 14 (1996), 51-60.

10. K. Kurosawa and Y. Desmedt, Optimum traitor tracing and asymmetric schemes, Advances in Cryptology: EUROCRYPT'98, Lecture Notes in Computer Science, 1403 (1998), 145-157.

11. M. Naor and B. Pinkas, Threshold traitor tracing, Advances in Cryptology: CRYPTO'98, Lecture Notes in Computer Science 1462 (1998), 502-517.

12. B. Pfitzmann, Trials of traced traitors, Information Hiding, Lecture Notes in Computer Science, 1174 (1996), 49-64.

collusions, In

13. J.N. Staddon, A combinatorial study of communication, storage and traceability in broadcast encryption systems, $\mathrm{PhD}$ thesis, University of California at Berkeley, 1997.

14. D.R. Stinson, An explication of secret sharing schemes, Designs, Codes and Cryptography, 2 (1992), 357-390.

15. D.R. Stinson, On some methods for unconditionally secure key distribution and broadcast encryption, Designs, Codes and Cryptography, 12 (1997), 215-243

16. D.R. Stinson and Tran van Trung, Some new results on key distribution patterns and broadcast encryption, Designs, Codes and Cryptography, 14 (1998), 261-279.

17. D.R. Stinson and R. Wei, Combinatorial properties and constructions of traceability schemes and frameproof codes, SIAM J. Discrete Math, 11 (1998), 41-53. 